\title{
Final LDRD Report for Feasibility Study
}

Tracking \#03-FS-005

R. I. Klein, B. Remington

February 12, 2004

U.S. Department of Energy

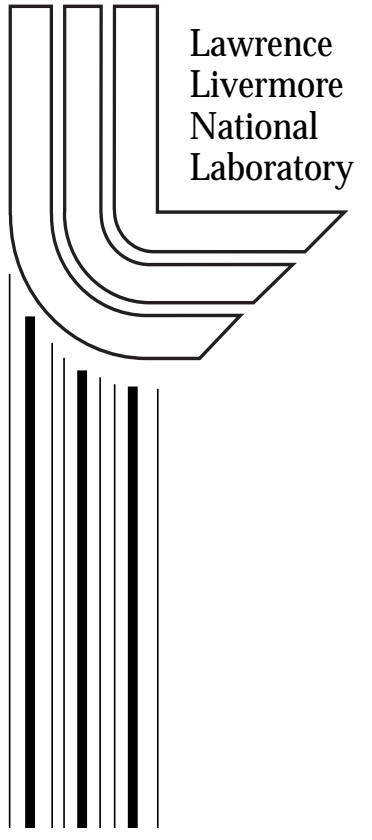


This document was prepared as an account of work sponsored by an agency of the United States Government. Neither the United States Government nor the University of California nor any of their employees, makes any warranty, express or implied, or assumes any legal liability or responsibility for the accuracy, completeness, or usefulness of any information, apparatus, product, or process disclosed, or represents that its use would not infringe privately owned rights. Reference herein to any specific commercial product, process, or service by trade name, trademark, manufacturer, or otherwise, does not necessarily constitute or imply its endorsement, recommendation, or favoring by the United States Government or the University of California. The views and opinions of authors expressed herein do not necessarily state or reflect those of the United States Government or the University of California, and shall not be used for advertising or product endorsement purposes.

This work was performed under the auspices of the U.S. Department of Energy by University of California, Lawrence Livermore National Laboratory under Contract W-7405-Eng-48. 


\section{Final LDRD Report for Feasibility Study}

PI Richard I. Klein

Co-PI Bruce Remington

Tracking number 03-FS-005

\section{Purpose:}

\section{Introduction:}

Extreme conditions of density and temperature of interest to DNT are similar to conditions of low-altitude atmospheres of neutron stars. Consequently, HED experimental capabilities being developed at LLNL (NIF, petawatt lasers) will open the door to laboratory studies of neutron star atmospheres. This capability will seed a new era in the study of extreme physics generated by strongly radiation dominated flows and laser-plasma interactions for the laboratory study of distant astrophysical phenomena. Indeed as has been noted by the recent Davidsen report on Frontiers of HEDPP (p. 85) "Accretion disks and atmospheres of neutron stars likely fall in the radiation-dominated regimes, where the radiation pressure dominates the particle pressure. Unique dynamics can ensue in such a radiation dominated plasma, especially in the presence of turbulent flows and magnetic fields. With the next generation of HED facilities such as ZR, NIF, coupled with ultra-intense laser "heater beams", it may become possible to create radiation-dominated plasma conditions in the laboratory relevant to neutron star (and black hole) accretion dynamics".

With the recent advent of the Rossi XTE time-resolved x-ray satellite, we have entered a new era in our ability to probe the physics and dynamics of neutron stars and black holes on rapid timescales not previously possible. With RXTE, we have diagnosed the dynamics occurring near the surface of a neutron star on timescales less than a millisecond, and have discovered a new phenomena, photon bubble instabilities, in an a accreting X-ray pulsar Centaurus X-3, some 30,000 light years across the galaxy. (Jerningan, Klein and Arons, ApJ, 2000) We in fact, predicted this instability in simulations with time-dependent $2 \mathrm{D}$ radiation-hydrodynamics codes that we had developed. (Klein et al. ApJ 1996a, 1996b) Strongly magnetized neutron stars accrete mass from a nearby normal star that is in orbit with the neutron star. The mass is transferred by way of an accretion disk and eventually finds its way onto the surface of the neutron star, where it is channeled by the strong magnetic fields (typically dipolar) onto polar caps that occupy a small surface $\left(\sim 1 \mathrm{~km}^{2}\right)$ area on the neutron star.

Photon Bubbles are a violent radiation-hydrodynamic instability whereby low density bubbles (buoyant with respect to the surrounding optically thick plasma flow) fill up with hot $10 \mathrm{keV}$ radiation, grow non-linearly and cause the plasma to become turbulent. The instability occurs when the radiation force on matter exceeds the 
gravitational force, a regime called super Eddington accretion. As has been shown with a linear stability analysis (Arons ApJ 1992), the low density regions in the midst of surrounding optically thick gas, experience a net flux of radiation and increase in buoyancy. If the magnetic field is appreciable ( $\mathrm{B}>10^{8} \mathrm{Gauss}$ ) a conductive increase in internal energy gives unstable growth with respect to the optically thick surrounding regions. This instability appears as an entropy mode in the accreting plasma. While some aspects of these flows are peculiar to the strongly magnetized neutron stars, most are not. Much of the phenomenology is expected in all super-Eddington flows, whether in accretion powered pulsars, low mass X-ray binaries or in the disks around black holes in active galactic nuclei.

The main purpose of our feasibility grant of $\$ 75,000$ for FY 2003 was to begin the study of the feasibility of generating, in a laboratory plasma, conditions that would mimic the conditions present in the low lying atmosphere of a magnetized neutron star that could potentially give rise to photon bubble instabilities, and eventually permit us to probe the physics of accreting, magnetized compact objects such as neutron stars and black holes. This would provide a unique way to explore some of the most exotic astrophysical phenomena in the universe, using powerful high energy density lasers such as NIF and petawatt laboratory platforms.

\section{Activities}

Using the feasibility grant of $\$ 75,000$, PI Richard Klein and Co-I Bruce Remington

began with engaging Steve Moon (PAT directorate) to work with us to address what conditions were needed to drive the photon bubble instability and to determine if scaled laboratory conditions could be achieved with upcoming laser facilities such as NIF and current petawatt lasers now present at Rutherford Laboratory in England.

To address these issues, we employed analytic techniques using linear stability analysis first used by us to study the linear growth development of photon bubble instabilities in neutron stars, and applied this analysis to determine rates of growth of this instability in a laboratory plasma.

With the feasibility grant this last year we have also made both PIC and hybrid code calculations to demonstrate the existence of ultra strong magnetic fields with ultra intense lasers and have made the first approximate calculations of the laboratory conditions needed to simulate the radiation dominated flow regime necessary to achieve neutron star atmosphere conditions and photon bubbles on NIF/Petawatt laser platforms.

With the feasibility grant we assembled a working group of first rate scientists within the laboratory to study with us the conditions needed to duplicate the phenomena on a neutrons star and delineate the various experimental and theoretical obstacles that must be overcome in order to achieve a successful set of laboratory experiments. This working group consisted of PI Richard Klein (DNT), Co-PI Bruce Remington (NIF), Steve Moon (PAT), Dimitri Ryutov (PAT), Scott Wilks (PAT), Andrew MacKinnon 
(NIF), Prav Patel (PAT), Leslie Rosenberg (NIF) and Anatoly Spitkovsky (UC Berkeley and Stanford University).

With this working group we met regularly every 2-3 weeks throughout 2003 to discuss progress. We were frequently joined by Garrett Jernigan and Jon Arons (UC Berkeley), Richard Towns (DNT) and Max Tabak (DNT).

\section{Technical Outcomes}

As a result of our feasibility studies during FY2003 we found that to achieve neutron star atmospheric conditions in a laboratory setting, we need radiation temperatures of order $1 \mathrm{keV}$ to achieve radiation pressure dominance, densities of order $\mathrm{n}_{\mathrm{e}} \sim 10^{22}$ in a high $\mathrm{Z}$ material to obtain appropriate optical depths $>1$, accelerations $>$ $10^{14} \mathrm{~cm}-\mathrm{s}^{-2}$ to provide an effective gravity, and magnetic field strengths $\sim 10^{8}$ Gauss that can confine the plasma to move in one direction. A stability analysis showed that the photon bubbles form when radiation conduction destabilizes the g-modes in the column, provided the magnetic field is strong enough to suppress the stabilizing expansion of the modes, and this occurs when the vertical magnetic field is $\mathrm{B} \sim 10^{8} \mathrm{G}$. Field strengths in excess of this have already been demonstrated by Tatarakis et al. at Imperial College group for a Petawatt laser. Our initial estimates show that relevant densities, accelerations and magnetic field strengths may be achievable on a petawatt class lasers coupled to NIF. Our recent feasibility simulations using a new hybrid PIC/fluids code (LSP) show that a 400 MegaGauss magnetic field inside the over dense plasma can be created, and fields $\sim 10^{8} \mathrm{G}$ can be transported well into a solid target. We also find that thermal temperatures of order $\sim 1 \mathrm{keV}$ are generated. The challenge for the design of this experiment will be to obtain radiation temperatures of the order of $1 \mathrm{keV}$ and an optical depth great enough in the plasma to achieve optically thick conditions. These conditions will be necessary to develop a strong radiation dominated flow which can generate the photon bubble instability, as well as keep the diffusion time of the plasma high enough to allow the plasma to stay radiatively hot long enough to have bubble growth occur for the duration of the experiment $\sim 2$ picoseconds. Radiation temperatures of $\sim 1 \mathrm{keV}$ may be achievable using NIF plus petawatt lasers. This will be further investigated by performing detailed PIC and hybrid (particles plus fluid) simulations. Our estimates show that bubble growth times are $\sim 0.5 \mathrm{ps}$, cooling times are $\sim 1.4 \mathrm{ps}$, laser pulse times $\sim 1-2$ ps, optical depths with high $\mathrm{Z}$ of a few; and effective gravities $\sim 10^{16} \mathrm{cms}^{-2}$.

Obtaining these high optical depths will be challenging, and several options looked promising. One possible high temperature approach is to use a holhraum to (1) heat and vertically expand a high $\mathrm{Z}$ target of several microns to lower its density and "build" the accretion column, then (2) turn up the hohlraum drive and use an ablatively driven shock to accelerate the column of high $\mathrm{Z}$ to an effective gravitational acceleration of $10^{16} \mathrm{~cm}-\mathrm{s}^{-2}$ and (3) use the petawatt laser as a split beam to impact the target on opposite sides to generate the appropriate magnetic field configurations to constrain the plasma flow to 1$\mathrm{D}$ and simultaneously heat the plasma column to $1 \mathrm{keV}$. Another promising approach uses larger targets (several $\mathrm{cm})$, requiring lower temperatures $(\sim 100 \mathrm{eV})$ to obtain higher opacities and optical depths, requiring smaller magnetic fields ( $1 \mathrm{MG})$, but large 
energies $(\sim 1 \mathrm{MJ})$ using all of NIF. Given our estimates, it may be possible to duplicate aspects of a neutron star atmosphere and possibly generate photon bubbles. 